\title{
ANÁLISE DO BANCO DE SEMENTES VISANDO ESTRATÉGIA DE TRANSPOSIÇÃO PARA A RESTAURAÇÃO ECOLÓGICA NO RIO GRANDE DO SUL
}

\author{
Bruna Balestrin Piaia ${ }^{1 *}$, Ana Paula Moreira Rovedder ${ }^{2}$, Maureen de Moraes Stefanello ${ }^{3}$, \\ Roselene Marostega Felker ${ }^{1}$, Eliara Marin Piazza ${ }^{4}$ \begin{abstract}
${ }^{2}$ Universidade Federal de Santa Maria, Departamento de Ciências Florestais, Campus Sede, Santa Maria, Rio Grande do Sul, Brasil anarovedder@gmail.com

${ }^{3}$ Universidade Federal de Santa Maria, Programa de Pós-graduação em Engenharia Agrícola, Santa Maria, Rio Grande do Sul, Brasil mmstefanello@gmail.com eliarapiazza@hotmail.com
\end{abstract} \\ ${ }^{1 *}$ Universidade Federal de Santa Maria, Programa de Pós-graduação em Engenharia Florestal, Núcleo de Estudos e Pesquisas em \\ Recuperação de Áreas Degradadas, Frederico Westphalen, Rio Grande do Sul, Brasil - brunabpiaia@ gmail.com; rosifelker@ yahoo.com.br \\ ${ }^{4}$ Universidade Federal de Santa Maria, Departamento de Tecnologia da Informação, Frederico Westphalen, Rio Grande do Sul, Brasil -
}

Recebido para publicação: 20/05/2016 - Aceito para publicação: 25/08/2017

\begin{abstract}
Resumo
A análise preliminar do banco de sementes pode auxiliar no planejamento e execução da estratégia de transposição e, assim, potencializar o sucesso da restauração ecológica. Objetivou-se avaliar a composição florística do banco de sementes em três fragmentos da Floresta Estacional na região central do Rio Grande do Sul. Os tratamentos foram: centro de fragmento florestal com formato retangular em estágio sucessional inicial a intermediário (FI), centro (FII-C) e borda (FII-B) de fragmento com formato circular em estágio sucessional inicial a intermediário e voçoroca estabilizada em sucessão inicial e formato alongado (FIII). Foram coletadas amostras até a profundidade de $5 \mathrm{~cm}$ com gabarito de $0,25 \times 0,25 \mathrm{~m}$, sendo oito repetições por tratamento. As plântulas emergentes foram contadas e identificadas. Os locais foram comparados quanto ao número de sementes germinadas, riqueza de espécies, forma de vida e origem pelo teste de KruskalWallis. Foi observado um total de 473 indivíduos, de 30 espécies e 13 famílias. A maior riqueza foi encontrada em FII-B (17 espécies). FIII apresentou o maior número de sementes germinadas $\left(576\right.$ ind. $\left./ \mathrm{m}^{2}\right)$, maior germinação de poáceas e maior densidade de exóticas invasoras. FI e FII-B apresentaram maior diversidade e baixo número de espécies exóticas invasoras. Conclui-se que a análise preliminar foi eficiente para identificar áreas favoráveis à doação para transposição do solo, os remanescentes de floresta estacional em estágio sucessional intermediário (FI e FII-B) podem ser indicados como doadores do banco de sementes, por apresentarem maior diversidade e baixo número de espécies exóticas invasoras.

Palavras-chave: Estágio sucessional; estrutura ecossistêmica; sementes germinadas.
\end{abstract}

\begin{abstract}
Analysis of the seed bank for a transposition strategy for ecological restoration in Rio Grande do Sul. Preliminary analysis of the seed bank can help in the planning and execution of the transposition strategie and, thus, potentiate the success of ecological restoration. We objective to evaluate the floristic composition of the seed bank in three fragments of the seasonal forest in the center of Rio Grande do Sul. The treatments were center of forest fragment with rectangular design in initial to intermediate succession stage (FI), center (FII-C) and border positions (FII-B) of fragment with circular design in initial to intermediate succession stage and stabilized gully in initial succession with elongated design (FIII). Samples were collected to $5 \mathrm{~cm}$ depth with frame of $0.25 \times 0.25 \mathrm{~m}$, eight repeats by treatments. Emerging seedlings were counted and identified. The sites were compared for the number of germinated seeds, species richness, lifeforms and origins by the Kruskal-Wallis test. A total of 473 individuals belonging to 30 species and 13 botany families were observed. The greatest richness was found in FII-B (17 spp). FIII had the highest number of germinated seeds $\left(576 \mathrm{ind} . / \mathrm{m}^{2}\right)$, higher germination of Poaceae and higher density of invasive exotics. FI and FII-B showed greater diversity and low number of invasive exotic species. We conclude which the preliminary analysis was efficient to identify areas favorable to donation for soil transposition, the seasonal forest remnants in the intermediate successional stage (FI and FII-B) may be indicated as donors of the seed bank, because they present greater diversity and low number of invasive alien species.

Keywords: Successional stage; ecosystem structure; germinated seeds.
\end{abstract}

FLORESTA, Curitiba, PR, v. 47, n. 3, p. 221 - 228, jul. / set. 2017.

Piaia, B. B. et al.

ISSN eletrônico 1982-4688

DOI: $10.5380 /$ rf.v47i1.46842 


\section{INTRODUÇÃO}

As florestas nativas no Rio Grande do Sul passaram por intensa descaracterização decorrente da atividade antrópica. O processo iniciou com o ciclo da araucária, aproximadamente em 1940 e se intensificou com o advento da modernização agrícola, principalmente no Planalto Meridional, metade norte do estado (MONTEIRO; BLAUTH, 2006). Esse histórico contribuiu para um cenário de fragmentação de habitats, perda da diversidade biológica e ameaça de extinção de espécies, estima-se que a Floresta Ombrófila Mista e a Floresta Estacional são as formações mais impactadas restando apenas 12,84\% e 17,97\%, respectivamente, de sua cobertura original (CORDEIRO; HASENACK, 2009).

Atualmente, existe uma carência de informações científicas quanto às especificidades dos ecossistemas em relação a alternativas de restauração ecológica na região sul do Brasil, pois esses são fortemente influenciados pelo clima subtropical. Há uma necessidade de se validar metodologias ecologicamente eficientes e, ao mesmo tempo, com menores custos (ROVEDDER et al., 2014).

Estratégias nucleadoras têm se mostrado promissoras, iniciando ou facilitando os processos de sucessão natural em diversas situações ambientais (CORBIN; HOLL, 2012). Entre as estratégias de nucleação, a transposição do banco de sementes consiste na retirada da camada superficial do solo de uma área doadora conservada e sua deposição em área degradada de mesma tipologia vegetal (REIS et al., 2014). O solo depositado na área degradada serve de fonte de propágulos para o recobrimento das áreas, elevando a abundância e riqueza de espécies, e estabelecendo novo ritmo sucessional na área degradada (HALL et al., 2010).

A transposição do solo visa aumentar a diversidade e variabilidade genética e elevar as chances de recrutamento de espécies adaptadas à condições adversas (REIS et al., 2014). Contudo, o banco de sementes pode conter espécies indesejáveis, como exóticas invasoras que podem inibir o processo de sucessão. A escolha de áreas doadoras deve ser baseada em critérios técnicos como análise do tamanho do fragmento, efeito de borda, histórico de distúrbios, presença de espécies exóticas invasoras. A análise preliminar da composição florística do banco de sementes pode auxiliar no planejamento e execução da técnica e potencializar o sucesso da restauração ecológica (MIRANDA NETO et al., 2010), servindo como um indicador do estado de conservação e potencial de regeneração após a transposição do solo.

Dessa forma, objetivou-se avaliar a composição florística do banco de sementes de fragmentos de floresta estacional, em diferentes estágios sucessionais, no Rio Grande do Sul, com vistas a identificar características favoráveis e possíveis limitações para a transposição do banco de sementes como estratégia de restauração ecológica em condições subtropicais.

\section{MATERIAL E MÉTODOS}

O estudo foi realizado em Santa Maria, região central do Rio Grande do Sul. A altitude média é de 103 m em relação ao nível do mar e o clima, segundo a classificação climática de Köppen é do tipo Cfa, subtropical úmido com verões quentes, sem estação seca definida. A temperatura média do mês mais frio é $13,3^{\circ} \mathrm{C}$, e a do mês mais quente é $24,5^{\circ} \mathrm{C}$; a precipitação pluviométrica média mensal oscila entre $134 \mathrm{~mm}$ e $192 \mathrm{~mm}$ (ALVARES et al., 2013).

O local do estudo situa-se na Depressão Periférica, extensa bacia sedimentar que se estende aproximadamente pelo centro do estado, no sentido leste-oeste. Os solos são derivados de arenitos, com estratificação de siltitos e argilitos. O relevo é suave-ondulado, predominando argissolos nas elevações e terço médio de encostas e planossolos e gleissolos nas cotas de menor altitude (STRECK et al., 2008). A região é zona de contato entre o Bioma Pampa e o Bioma Mata Atlântica, sendo que a formação florestal característica é a Floresta Estacional (FE).

Os fragmentos florestais do estudo se encontram em matriz de produção agrícola, possuindo no entorno pastagens e culturas anuais como a soja. Definiram-se os seguintes tratamentos:

- $\quad$ Fragmento I (FI): remanescente de FE em estágio sucessional inicial a intermediário, com espécies pioneiras e secundárias iniciais e altura média do dossel de $8,5 \mathrm{~m}$. Possui formato retangular e área de 25 ha. Neste remanescente, retiraram-se amostras do centro do fragmento (distância $\geq$ a $50 \mathrm{~m}$ da bordadura da floresta);

- $\quad$ Fragmento II-C (FII-C): remanescente de FE em estágio sucessional inicial a intermediário, apresentando espécies pioneiras e secundárias iniciais e altura média do dossel de $12 \mathrm{~m}$. Possui área de 30 ha e formato circular. Foram retiradas amostras do centro do fragmento (distância $\geq$ a $50 \mathrm{~m}$ da bordadura da floresta);

- Fragmento II-B (FII-B): o mesmo remanescente anterior, porém, retiraram-se amostras da borda do fragmento (distância $\leq$ a $10 \mathrm{~m}$ da bordadura da floresta);

- $\quad$ Fragmento III (FIII): voçoroca estabilizada, em estágio de sucessão inicial, com espécies pioneiras e altura média do dossel de $4 \mathrm{~m}$. Possui 2 ha e formato alongado, com efeito de borda em toda sua extensão. 
A coleta do banco de sementes foi realizada com gabarito de $0,25 \mathrm{~m} \mathrm{x} 0,25 \mathrm{~m}$ e profundidade de $5 \mathrm{~cm}$. A amostragem foi sistematizada, com distância de $20 \mathrm{~m}$ entre cada ponto de coleta e oito repetições por tratamento. Cada amostra foi armazenada em saco plástico e transportada até a área experimental do Departamento de Ciências Florestais, no campus sede da Universidade Federal de Santa Maria, onde foram colocadas em bandejas de 0,50 m x 0,30 m x 0,05 m, com uma camada de vermiculita na base. As bandejas foram dispostas em canteiros a pleno sol para simular a condição de luminosidade após transposição e receberam regas diárias.

As avaliações foram realizadas mensamente, iniciaram em setembro de 2013 e seguiram até janeiro de 2014, quando a germinação de novas sementes cessou. As plântulas que emergiram foram contadas, identificadas e removidas das bandejas no momento da avaliação. As plântulas não identificadas foram transplantadas para posterior identificação em fase de maior desenvolvimento. A identificação das espécies foi realizada com ajuda de literatura e consulta à especialistas. Adotou-se o sistema de classificação APG III (2009), e os nomes das espécies foram confirmados por meio do site do Missouri Botanical Garden.

Foram calculados o índice de diversidade de Shannon, equabilidade pelo índice de Pielou e similaridade florística pelo índice de Jaccard (MAGURRAN, 2013). A densidade de plântulas foi extrapolada para um metro quadrado. As espécies foram classificadas quanto à origem, em exótica e nativa regional com base na distribuição das espécies, forma de vida e síndromes de polinização (FAEGRI; PIJL, 1979) e dispersão (PIJL, 1982). Os indivíduos da família Poaceae foram separados em um grupo independente, devido à representatividade no banco de sementes. Sendo assim, a categoria denominada "herbáceas" ao longo do texto se refere aos indivíduos dessa forma de vida de todas as famílias botânicas encontradas, com exceção de Poaceae. E a categoria denominada "gramíneas" refere-se, exclusivamente, às poáceas herbáceas.

As análises estatísticas foram feitas no programa R (R DEVELOPMENT CORE TEAM, 2016). O número de sementes germinadas, riqueza de espécies e densidade por forma de vida, origem e síndromes de polinização e dispersão foram submetidos aos testes de Kolgomorov-Smirnov, para verificar a normalidade, e Bartlett, para a homogeneidade de variância. Esses pressupostos não foram atendidos, portanto utilizou-se estatística não paramétrica.

Os tratamentos foram comparados quanto ao número de sementes germinadas, densidade por forma de vida, riqueza de espécies total e em relação à origem, síndromes de polinização e dispersão pelo teste de Kruskal-Wallis $(\mathrm{p}<0,05)$. Quando houve diferença significativa entre os tratamentos, a média dos postos foi comparada pelo teste $\mathrm{t}(\mathrm{p}<0,05)$, de acordo com o pacote agricolae do programa R (MENDIBURU, 2015).

Os valores do índice de diversidade de Shannon foram comparados pelo teste-t de Hutcheson, ao nível de $5 \%$ de probabilidade $(\mathrm{p}<0,05)$, com comparações feitas aos pares (HUTCHESON, 1970).

\section{RESULTADOS}

Observou-se um total de 473 sementes germinadas, pertencentes a 13 famílias botânicas, 28 gêneros e 30 espécies. Destas, uma foi identificada apenas em nível de família e duas não foram identificadas $(6,7 \%)$. A família Asteraceae foi a mais representativa (9 spp.), seguida de Poaceae (3 spp.) e Solanaceae (3 spp.). As espécies que apresentaram maior número de sementes germinadas foram Solanum americanum Mill., Actinostemon concolor (Spreng.) Müll.Arg. e Axonopus sp. no banco de sementes de FI, Axonopus sp., Paspalum sp. e Solanum americanum Mill. em FII-C, Paspalum sp., Amaranthus hybridus e Vernonanthura tweedieana (Baker) H. Rob. em FII-B e Cyperus sp., Urochloa decumbens Stapf e Senegalia bonariensis (Gillies ex Hook. \& Arn.) Seigler \& Ebinger em FIII (Tabela 1). Quanto à forma de vida, predominaram herbáceas $(73,3 \%)$ seguidas de arbóreas $(16,7 \%)$, arbustivas $(6,7 \%)$ e trepadeiras $(3,3 \%)$ (Tabela 1$)$.

Tabela 1. Número de sementes germinadas por espécie e suas características de forma de vida (FV), origem (O), síndrome de polinização (SP) e dispersão (SD) no banco de sementes dos tratamentos analisados (FI, FII-C, FII-B e FIII), Santa Maria, Rio Grande do Sul.

Table 1 Number of germinated seeds by species and their characteristics lifeform (FV), origin (O), pollination (SP) and dispersion (SD) syndrome in the seed bank of treatments analyzed (FI, FII-C, FII-B e FIII), Santa Maria, Rio Grande do Sul.

\begin{tabular}{|c|c|c|c|c|c|c|c|c|c|}
\hline \multirow{2}{*}{ Família/Espécie } & \multirow{2}{*}{ FV } & \multirow{2}{*}{$\mathbf{O}$} & \multirow{2}{*}{ SP } & \multirow{2}{*}{ SD } & \multicolumn{5}{|c|}{ Número de sementes germinadas } \\
\hline & & & & & FI & FII-C & FII-B & FIII & Total \\
\hline Amaranthaceae & & & & & & & & & \\
\hline $\begin{array}{l}\text { Amaranthus hybridus L. } \\
\text { Aralicaceae }\end{array}$ & her & $\mathrm{e}$ & zoof & anec & & & 11 & 23 & 34 \\
\hline $\begin{array}{l}\text { Hydrocotyle sp. } \\
\text { Asteraceae }\end{array}$ & her & $\mathrm{n}$ & zoof & aut & & & 1 & & 1 \\
\hline Baccharis dracunculifolia DC. & Arb & $\mathrm{n}$ & zoof & anec & & & 1 & & 1 \\
\hline
\end{tabular}

FLORESTA, Curitiba, PR, v. 47, n. 3, p. 221 - 228, jul. / set. 2017

Piaia, B. B. et al.

ISSN eletrônico 1982-4688

DOI: $10.5380 /$ rf.v47i1.46842 


\begin{tabular}{|c|c|c|c|c|c|c|c|c|c|}
\hline Chaptalia sp. & her & $\mathrm{n}$ & zoof & anec & 2 & 1 & & 1 & 4 \\
\hline Conyza sp. & her & $\mathrm{n}$ & zoof & anec & 1 & & & & 1 \\
\hline Hypochaeris sp. & her & $\mathrm{n}$ & zoof & anec & & 2 & 1 & & 3 \\
\hline Senecio brasiliensis (Spreng.) Less. & her & $\mathrm{n}$ & zoof & anec & 1 & 2 & 1 & & 4 \\
\hline Sonchus oleraceus L. & her & $\mathrm{e}$ & zoof & anec & 5 & 4 & 3 & & 12 \\
\hline Vernonanthura tweedieana (Baker) H. Rob. & arb & $\mathrm{n}$ & zoof & anec & & & 8 & & 8 \\
\hline Gamochaeta pensylvanica (Wild.) Cabrera & her & $\mathrm{n}$ & zoof & anec & & 1 & & & 1 \\
\hline Morfoespécie 4 & her & nd & zoof & anec & 1 & 3 & & & 4 \\
\hline \multicolumn{10}{|l|}{ Cyperaceae } \\
\hline Bulbostylis capillaris (L.) C.B. Clarke & her & $\mathrm{n}$ & anef & anec & & & 3 & 2 & 5 \\
\hline Cyperus sp. & her & $\mathrm{n}$ & anef & anec & & & & 64 & 64 \\
\hline \multicolumn{10}{|l|}{ Euphorbiaceae } \\
\hline $\begin{array}{l}\text { Actinostemon concolor (Spreng.) Müll.Arg. } \\
\text { Fabaceae }\end{array}$ & árv & $\mathrm{n}$ & zoof & aut & 8 & & & & 8 \\
\hline Desmodium sp. & her & $\mathrm{n}$ & zoof & zooc & 1 & & & 1 & 2 \\
\hline $\begin{array}{l}\text { Senegalia bonariensis (Gillies ex Hook. \& } \\
\text { Arn.) Seigler \& Ebinger } \\
\text { Malvaceae }\end{array}$ & $\mathrm{arb} / \mathrm{tr}$ & $\mathrm{n}$ & zoof & anec & & & 3 & 47 & 50 \\
\hline $\begin{array}{l}\text { Sida sp. } \\
\text { Molluginaceae }\end{array}$ & her & $\mathrm{n}$ & zoof & zooc & & & 1 & 1 & 2 \\
\hline $\begin{array}{l}\text { Mollugo verticillata } \mathrm{L} \text {. } \\
\text { Poaceae }\end{array}$ & her & $\mathrm{n}$ & zoof & aut & & 1 & & & 1 \\
\hline Axonopus sp. & her & $\mathrm{n}$ & anef & anec & 7 & 33 & 5 & & 45 \\
\hline Urochloa decumbens Stapf & her & $\mathrm{e}$ & anef & anec & & & 6 & 57 & 63 \\
\hline $\begin{array}{l}\text { Paspalum sp. } \\
\text { Portulacaceae }\end{array}$ & her & $\mathrm{n}$ & anef & anec & 3 & 9 & 15 & 38 & 65 \\
\hline $\begin{array}{l}\text { Portulaca oleracea } \mathrm{L} \text {. } \\
\text { Rutaceae }\end{array}$ & her & $\mathrm{e}$ & zoof & anec & & & & 3 & 3 \\
\hline Zanthoxylum sp. & Árv & $\mathrm{n}$ & zoof & zooc & & & 2 & & 2 \\
\hline & \multicolumn{6}{|c|}{ Salicaceae } & 2 & & 2 \\
\hline \multicolumn{9}{|l|}{ Solanaceae } & 1 \\
\hline Solanum americanum Mill. & her & $\mathrm{n}$ & zoof & zooc & 13 & 4 & 5 & 44 & 66 \\
\hline Solanum mauritianum Scop. & Árv & $\mathrm{n}$ & zoof & zooc & 5 & 2 & 1 & & 8 \\
\hline $\begin{array}{l}\text { Solanum viarum Dunal } \\
\text { Não determinadas }\end{array}$ & her & $\mathrm{n}$ & zoof & zooc & & 2 & & 6 & 8 \\
\hline Morfoespécie 1 & her & nd & nd & nd & 3 & 1 & & & 4 \\
\hline Morfoespécie 2 & her & nd & nd & nd & & & & 1 & 1 \\
\hline Total geral & & & & & 51 & 65 & 69 & 288 & 473 \\
\hline
\end{tabular}

Em que: her: herbácea; subar: subarbustiva; arb: arbustiva; árv: arbórea; tr: trepadeira; nd: não determinado; n: nativa; e: exótica; anef: anemofílica; zoof: zoofílica; anec: anemocórica; zooc: zoocórica; aut: autocórica.

A forma de vida herbácea foi dominante em todos os locais analisados (Tabela 2), destacando-se as da família Poaceae, observando-se valor mais expressivo em FIII, com densidade elevada de Urochloa decumbens.

Tabela 2. Densidade de indivíduos $/ \mathrm{m}^{2}$ por forma de vida encontrados no banco de sementes dos tratamentos analisados (F, FII-C, FII-B e FIII), Santa Maria, Rio Grande do Sul.

Table 2. Individuals $/ \mathrm{m}^{2}$ density by lifeform found in the seed bank of the treatments analyzed (F, FII-C, FII-B e FIII), Santa Maria, Rio Grande do Sul.

\begin{tabular}{cccccc}
\hline \multirow{2}{*}{ Local } & \multicolumn{5}{c}{ Forma de vida } \\
\cline { 2 - 5 } & Herbácea* & Gramíneas & Arbustiva & Arbórea & Trepadeira \\
\hline FI & $54 \mathrm{~b}^{* *}$ & $18 \mathrm{~b}$ & $0 \mathrm{~b}$ & $28 \mathrm{a}$ & $0 \mathrm{~b}$ \\
FII-C & $40 \mathrm{~b}$ & $86 \mathrm{ab}$ & $0 \mathrm{~b}$ & $4 \mathrm{~b}$ & $0 \mathrm{~b}$ \\
FII-B & $52 \mathrm{~b}$ & $52 \mathrm{ab}$ & $18 \mathrm{a}$ & $10 \mathrm{ab}$ & $6 \mathrm{~b}$ \\
FIII & $294 \mathrm{a}$ & $184 \mathrm{a}$ & $0 \mathrm{~b}$ & $0 \mathrm{~b}$ & $94 \mathrm{a}$ \\
*A classe herbáceas se refere a todos os indivíduos dessa forma de vida, exceto aos da família Poaceae. Esses estão inclusos na categoria "gramíneas". \\
**Médias seguidas pela mesma letra, na coluna, não diferem entre si pelo teste de Kruskal-Wallis (p<0,05). \\
\hline
\end{tabular}


Em relação à densidade de espécies arbóreas, os maiores valores foram obtidos para FI, seguido por FIIB (Tabela 2). Foram identificadas cinco espécies: Actinostemon concolor (Spreng.) Müll.Arg, Banara tomentosa Clos, Helietta apiculata Benth., Solanum mauritianum Scop. e Zanthoxylum sp., sendo que A. concolor pode ser considerada de sub-bosque, B. tomentosa, secundária tardia, Helietta apiculata, Zanthoxylum sp. e $S$. mauritianum, de estágios iniciais de sucessão. Em FIII não houve germinação de espécies arbóreas.

Quanto à origem, 76,7\% são nativas, 13,3\% são exóticas e 10,0\%, não foram determinadas. As espécies exóticas ocorreram em todos os tratamentos, com valores superiores em FII-B e FIII (Tabela 3). A síndrome de polinização zoofílica predominou $(76,7 \%)$ em relação à anemofílica $(16,7 \%)$. Para $6,6 \%$ das espécies não foi possível a determinação. A polinização anemofílica foi superior em FIII, já a zoofilica não apresentou diferença significativa entre os locais estudados (Tabela 3). Em relação à síndrome de dispersão, 60,0\% das espécies são anemocóricas, $23,3 \%$ zoocóricas, $10,00 \%$ autocóricas e $6,7 \%$ não foram determinadas. Houve diferença significativa entre os tratamentos para a síndrome de dispersão anemocórica, que foi superior em FIII. FI destacouse quanto à autocoria e para as espécies com síndrome de dispersão zoocórica não houve diferença (Tabela 3).

Tabela 3. Riqueza de espécies germinadas no banco de semente dos tratamentos avaliados (FI, FII-C, FII-B, FIII), em relação à origem, síndromes de polinização e dispersão, em Santa Maria, Rio Grande do Sul.

Table 3. Species richness germinated in the seed bank of the evaluated treatments (FI, FII-C, B-FII, FIII) in relation to origin, pollination and dispersal syndromes, Santa Maria, Rio Grande do Sul.

\begin{tabular}{ccccccccccc}
\hline \multirow{2}{*}{ Trat. } & \multicolumn{3}{c}{ Origem } & \multicolumn{4}{c}{ Síndromes de Polinização } & \multicolumn{3}{c}{ Síndromes de Dispersão } \\
\cline { 2 - 10 } & Exót. & Nat. & Nd. & Anef. & Zoof. & Nd. & Anec. & Aut. & Zooc. & Nd. \\
\hline FI & $0,4 \mathrm{~b}$ & $3,0 \mathrm{~b}$ & $0,5 \mathrm{a}$ & $0,6 \mathrm{~b}$ & $2,9 \mathrm{a}$ & $0,4 \mathrm{a}$ & $1,6 \mathrm{c}$ & $0,6 \mathrm{a}$ & $1,3 \mathrm{a}$ & $0,4 \mathrm{a}$ \\
FII-C & $0,5 \mathrm{~b}$ & $2,1 \mathrm{~b}$ & $0,5 \mathrm{a}$ & $0,5 \mathrm{~b}$ & $2,5 \mathrm{a}$ & $0,1 \mathrm{ab}$ & $2,1 \mathrm{bc}$ & $0,1 \mathrm{~b}$ & $0,8 \mathrm{a}$ & $0,1 \mathrm{ab}$ \\
FII-B & $1,3 \mathrm{a}$ & $2,3 \mathrm{~b}$ & $0,0 \mathrm{a}$ & $0,9 \mathrm{~b}$ & $2,6 \mathrm{a}$ & $0,0 \mathrm{~b}$ & $2,8 \mathrm{~b}$ & $0,1 \mathrm{~b}$ & $0,6 \mathrm{a}$ & $0,0 \mathrm{~b}$ \\
FIII & $1,6 \mathrm{a}$ & $4,1 \mathrm{a}$ & $0,1 \mathrm{a}$ & $2,5 \mathrm{a}$ & $3,3 \mathrm{a}$ & $0,1 \mathrm{ab}$ & $4,5 \mathrm{a}$ & $0,0 \mathrm{~b}$ & $1,3 \mathrm{a}$ & $0,1 \mathrm{ab}$ \\
\hline
\end{tabular}

Em que: Nat: nativa; Exót: exótica; Nd: não determinado; Anef: anemofílica; Zoof: zoofílica; Anec: anemocórica; Aut: autocórica; Zooc: zoocórica.

*Médias seguidas pela mesma letra, na coluna, não diferem entre si pelo teste de Kruskal-wallis $(\mathrm{p}<0,05)$.

Não houve diferença significativa entre FI, FII-C e FII-B para o número de sementes germinadas e de espécies, contudo a área FIII diferiu das demais, apresentando valor superior de sementes germinadas e riqueza de espécies (Tabela 4). A diversidade expressa pelo índice de Shannon não apresentou diferença significativa entre os locais pelo teste de Hutcheson ao nível de 5\% de probabilidade (Tabela 4).

Tabela 4. Densidade, riqueza e diversidade das amostras do banco de sementes dos tratamentos avaliados (FI, FII-C, FII-B e FIII), em Santa Maria, Rio Grande do Sul.

Table 4. Density, richness and diversity of seed bank samples from evaluated treatments (FI, FII-C, FII-B e FIII), em Santa Maria, Rio Grande do Sul.

\begin{tabular}{|c|c|c|c|c|}
\hline Variável & FI & FII-C & FII-B & FIII \\
\hline $\mathrm{N}^{\mathrm{o}}$ sementes germinadas $/ \mathrm{m}^{2}$ & $102 b^{*}$ & $130 \mathrm{~b}$ & $138 \mathrm{~b}$ & $576 a$ \\
\hline $\mathrm{N}^{\mathrm{o}}$ de espécies & $3,9 b^{*}$ & $3,1 b$ & $3,5 b$ & $5,9 \mathrm{a}$ \\
\hline $\mathrm{N}^{\mathrm{o}}$ total de espécies & 13 & 13 & 17 & 13 \\
\hline Índice de diversidade de Shannon $\left(\mathrm{H}^{\prime}\right)$ & $2,21 \mathrm{a}^{* *}$ & $1,79 \mathrm{a}$ & $2,35 \mathrm{a}$ & $1,95 \mathrm{a}$ \\
\hline Índice de equabilidade de Pielou $\left(\mathrm{J}^{\prime}\right)$ & 0,86 & 0,70 & 0,83 & 0,76 \\
\hline
\end{tabular}

"Médias seguidas pela mesma letra, na linha, não diferem entre si pelo teste de Kruskal-Wallis ( $\mathrm{p}<0,05)$.

** Índice de Shannon seguido pela mesma letra não diferiu pelo teste-t de Hutcheson $(\mathrm{p}<0,05)$.

O índice de similaridade de Jaccard variou entre 0,13 a 0,53 na interação entre os locais avaliados, o que demonstra baixa similaridade florística da composição do banco de sementes (Tabela 5).

Tabela 5. Similaridade pelo índice de Jaccard entre a composição de espécies do banco de sementes dos tratamentos analisados (FI, FII-C, FII-B e FIII), em Santa Maria, Rio Grande do Sul.

Table 5. Jaccard similarity index between the species composition of seed bank of evaluated treatments (FI, FII-C, FII-B e FIII), Santa Maria, Rio Grande do Sul.

\begin{tabular}{ccccc}
\hline Tratamentos & FI & FII-C & FII-B & FIII \\
\hline I & - & 0,53 & 0,25 & 0,18 \\
II-C & - & - & 0,30 & 0,13 \\
II-B & - & - & - & 0,30 \\
III & - & - & - & - \\
\hline
\end{tabular}

FLORESTA, Curitiba, PR, v. 47, n. 3, p. 221 - 228, jul. / set. 2017.

Piaia, B. B. et al.

ISSN eletrônico 1982-4688

DOI: $10.5380 /$ rf.v47i1.46842 


\section{DISCUSSÃO}

É possível observar a representatividade de Poaceae, Asteraceae e Solanaceae no banco de sementes, o que concorda com pesquisas que também analisaram banco de sementes da floresta estacional na região do estudo (SCOTTI et al., 2011) e em outras formações, como na Floresta Estacional Semidecidual na região sudeste (CALEGARI et al., 2013) e na Floresta Ombrófila Mista, em Santa Catarina (SCHORN et al., 2013). Em sua maioria, as espécies dessas famílias são herbáceas e pioneiras, produzem elevada densidade de diásporos e formam banco de sementes persistente (LONG et al., 2015).

A espécie exótica Urochloa decumbens é uma invasora agressiva em território brasileiro, apresenta efeito alelopático, o que confere maior poder competitivo (BARBOSA et al., 2008). Correia e Martins (2015) avaliaram o banco de sementes de uma floresta em restauração há 23 anos e de uma área de referência conservada no Espírito Santo, em Floresta Ombrófila Densa, e observaram elevada densidade de germinação de $U$. decumbens na área em restauração, apesar de não verificarem o estabelecimento da espécie devido ao dossel fechado. Os autores ressaltam a possível colonização, caso haja a abertura de clareira ou passagem do fogo, o que impediria a cicatrização por nativas de estágios iniciais da sucessão.

No Rio Grande do Sul, as baixas temperaturas do inverno reduzem o potencial de invasão biológica por $U$. decumbens, o que pode explicar porque essa espécie não é tão agressiva como nos estados do Brasil tropical. Os resultados do presente trabalho, contudo, demonstram que a espécie poderá expressar seu potencial invasor a partir de sua permanência no banco de sementes. $\mathrm{O}$ fato de a matriz produtiva regional ser predominantemente agropecuária também favorece sua presença. A maior densidade em FIII demonstra a relação entre estágio sucessional e potencial de invasão por espécies exóticas, com a modificação do microclima em áreas alteradas.

A maior densidade de herbáceas observada em todos os tratamentos pode estar relacionada ao ciclo de vida destas espécies, a alta produtividade de sementes, à proximidade com áreas de produção agrícola e pastagens vizinhas, que funcionam como fontes de propágulos. Excetuando-se os problemas com espécies invasoras, herbáceas são importantes nas áreas em processo de restauração, atuando como colonizadoras iniciais. Quando recrutadas têm o papel de modificar o ambiente e permitir uma nova dinâmica sucessional (TRES; REIS, 2009).

Portanto, na transposição do banco de sementes, a presença de herbáceas pode ser benéfica, desde que não sejam espécies exóticas invasoras, que, devido ao seu forte poder competitivo, podem suprimir a sucessão local. Espécies herbáceas nativas encontradas nos tratamentos do presente estudo podem auxiliar na colonização de substratos degradados, atuar na atração da fauna polinizadora e dispersora e contribuir para a melhoria da qualidade do solo (TRES; REIS, 2009). A inserção de diferentes formas de vida vegetal em projetos de restauração remete à diversidade de espécies, fundamental no restabelecimento dos processos ecológicos para garantir a continuidade nos ecossistemas (CORBIN; HOLL, 2012). A ausência de indivíduos arbóreos em FIII evidencia as consequências do histórico e intensidade de distúrbio, o que gera uma forma de retroalimentação em relação ao formato e estrutura ecossistêmica desta área.

A maior expressão de anemofilia e anemocoria em FIII, em relação às demais áreas, pode ser explicada pelo caráter de sucessão ecológica inicial, relacionado ao seu histórico (voçoroca estabilizada). O formato alongado, com intenso efeito de borda favorece o desenvolvimento de espécies de caráter pioneiro e a chegada de propágulos por anemocoria, que passam a fazer parte do banco de sementes. É sabido que as síndromes de anemofilia e anemocoria predominam nesse grupo de espécies vegetais (PIJL, 1982; FAEGRI; PIJL, 1979).

Espécies com síndrome de dispersão anemocórica e polinização anemofílica são importantes em projetos de restauração ecológica por atuarem como colonizadoras iniciais do ambiente degradado, não precisando de agentes dispersores e polinizadores. Por outro lado, espécies que apresentam interação com a fauna atuam como facilitadoras da sucessão ecológica. Essas favorecem o fluxo gênico, servem de alimento para a fauna dispersora e promovem a continuidade do processo sucessional da área (CORBIN; HOLL, 2012).

O número de sementes germinadas com valor superior em FIII deve-se ao maior número de herbáceas encontrado no banco de sementes desse local. A densidade do banco de sementes relaciona-se com o uso anterior do solo, grau de conservação da vegetação e bioma, mas tende a ser maior em florestas secundárias, em que o dossel mais aberto possibilita maior densidade de espécies pioneiras, portanto, a densidade do banco de sementes é variável conforme a tipologia e matriz vegetacional, histórico de exploração, usos do solo no entorno, distância de fontes de propágulos e intensidade da perturbação (BAIDER et al., 1999).

A heterogeneidade deve ser levada em consideração na transposição do banco de sementes, uma vez que eleva as chances de recrutamento de espécies adaptadas à condições diversas (TRES; REIS, 2009). Portanto, a diferença da composição florística entre as áreas pode aumentar a riqueza de espécies após a transposição do solo. 


\section{CONCLUSÕES}

- A análise do banco de sementes é eficiente para identificar características favoráveis que permitam a indicação da área como potencialmente doadora em estratégias de transposição do banco de sementes.

- A posição de coleta do banco de sementes no fragmento florestal (borda ou centro) e o estágio sucessional influenciam na composição do banco de sementes do solo.

- O estágio sucessional foi o fator preponderante para a presença de gramíneas invasoras no banco de sementes.

- As principais características indesejáveis encontradas e que se traduzem como fatores limitantes à transposição do banco de sementes são a presença de poáceas herbáceas e espécies exóticas invasoras. Dessa forma, a área em sucessão inicial (FIII) não foi indicada.

- Os remanescentes de floresta estacional em estágio sucessional intermediário (FI e FII-B) podem ser indicados como doadores do banco de sementes, por apresentarem maior diversidade e baixo número de espécies exóticas invasoras, o que caracteriza o menor nível de distúrbio.

\section{REFERÊNCIAS}

ALVARES, C. A.; STAPE, J. L.; SENTELHAS, P. C.; GONÇALVES, J. L. DE M.; SPAROVEK, G. Köppen's climate classification map for Brazil. Meteorologische Zeitschrift, Stuttgart, v. 22, n. 6, p. 711-728, 2013.

APG III. Angiosperm Phylogeny Group. An update of the Angiosperm Phylogeny Group classification for the orders and families of flowering plants: APG III. Botanical Journal of the Linnean Society, London, v. 161, n. 2, p. 105-121, 2009.

BAIDER, C.; TABARELLI, M.; MANTOVANI, W. O banco de sementes de um trecho de uma Floresta Atlântica Montana (São Paulo - Brasil). Revista Brasileira de Biologia, São Carlos, v. 59, n. 2, p. 319-328, 1999.

BARBOSA, E. G.; PIVELLO, V. R.; MEIRELLES, S. T. Allelopathic evidence in Brachiaria decumbens and its potential to invade the Brazilian cerrados. Brazilian Archives of Biology and Technology, Curitiba, v. 51, n. 4, p. 825-831, 2008.

CALEGARI, L.; MARTINS, S. V.; CAMPOS, L. C.; SILVA, E.; GLERIANI, J. M. Avaliação do banco de sementes do solo para fins de restauração florestal em Carandaí, MG. Revista Árvore, Viçosa, v. 37, n. 5, p. 871-880, 2013.

CORBIN, J. D.; HOLL, K. D. Applied nucleation as a forest restoration strategy. Forest Ecology and Management, Amsterdam, v. 265, p. 37-46, 2012.

CORDEIRO, J. L. P.; HASENACK, H. Cobertura vegetal atual do Rio Grande do Sul. In: PILLAR, V. de P.; MÜlleR, S. C.; CASTILHOS, Z. M. de S.; JACQUES A. V. A. Campos Sulinos: conservação e uso sustentável da biodiversidade. Brasília: MMA, 2009. 403p.

CORREIA, G. G. S.; MARTINS, S. V. Banco de Sementes do Solo de Floresta Restaurada, Reserva Natural Vale, ES. Floresta e Ambiente, Rio de Janeiro, v. 22, n. 1, p. 79-87, 2015.

FAEGRI, K.; van der PIJL, L. The principles of pollination ecology. Oxford: Pergamon Press, 1979. 244p.

HALL, S. L.; BARTON, C. D.; BASKIN, C. C. Topsoil seed bank of an Oak-Hickory forest in eastern Kentucky as a restoration tool on surface mines. Restoration Ecology, Oxford, v. 18, n. 6, p. 834-842, 2010.

HUTCHESON, K. A test for comparing diversities based on the Shannon formula. Journal of Theoretical Biology, London, v. 29, p. 151-154, 1970.

LONG, R. L.; GORECKI, M. J.; RENTON, M., SCOTT, J. K.; COLVILlE, L.; GOGGIN, D. E.; COMMANDER, L. E.; WESTCOTT, D. A,; CHERRY, H.; FINCH-SAVAGE, W. E. The ecophysiology of seed persistence: a mechanistic view of the journey to germination or demise. Biological Reviews, London, v. 90, p. 31-59, 2015.

MAGURRAN, A. E. Medindo a diversidade biológica. Tradução Dana Moina Vianna. Editora UFPR. 2013. 261p.

MENDIBURU, F de Package 'agricolae'. R package version 1.2-1 http://cran.r-project.org/web/packages/ agricolae. 2015.

FLORESTA, Curitiba, PR, v. 47, n. 3, p. 221 - 228, jul. / set. 2017

Piaia, B. B. et al.

ISSN eletrônico 1982-4688

DOI: $10.5380 /$ rf.v47i1.46842 
MIRANDA NETO, A.; KUNZ, S. H.; MARTINS, S. V.; SILVA, K. de A.; SILVA, D. A. da. Transposição do banco de sementes do solo como metodologia de restauração florestal de pastagem abandonada em Viçosa, MG. Viçosa -MG, Brasil. Revista Árvore, Viçosa, v. 3, n. 6, p. 1035-1043, 2010.

MISSOURI BOTANICAL GARDEN - MOBOT. Explore the beta release of web TROPICOS. Disponível em: <http://www.tropicos.org/> Acesso em: 18/01/2016.

MONTEIRO, K, V.; BLAUTH, N. Os estados da Mata Atlântica: Rio Grande do Sul. In: CAMPANILI, M.; PROCHNOW, M. Mata atlântica - uma rede pela floresta. Brasília: RMA, 2006. 334p.

PIJL, L. Principles of dispersal in higer plants. Berlin: Springer-Verlag, 1982. 162p.

R DEVELOPMENT CORE TEAM R: A language and environment for statistical computing. R Foundation for Statistical Computing, Vienna, Austria. 2013. ISBN 3-900051-07-0, Disponível em: http://www.R-project.org. http://web.mit.edu/r_v3.0.1/fullrefman.pdf. Acesso em: 04 nov 2014.

REIS, A.; BECHARA, F. C.; TRES, D. R.; TRENTIN, B. E. Nucleação: concepção biocêntrica para a restauração ecológica. Ciência Florestal, Santa Maria, v. 24, n. 2, p. 509-518, abr./jun. 2014.

ROVEDDER, A. P. M.; PIAIA, B. B.; FELKER, R. M.; PIAZZA, E. M.; HUMMEL, R. B. Perspectivas da restauração ecológica de ecossistemas para o Rio Grande do Sul. In: DÖRR, A. C.; ROSSATO, M. V.; ROVEDDER, A. P. M.; PIAIA, B. B. (Org.) Práticas e saberes em meio ambiente. Curitiba, Appris, 2014. 360p.

SCCOTI, M. S. V.; ARAUJO, M. M.; WENDLER, C. F. W.; LONGHI, S. J. Mecanismos de regeneração natural em remanescente de floresta estacional decidual. Ciência Florestal, Santa Maria, v. 21, n. 3, p. 455-468, 2011.

SCHORN, L. A.; FENILli, T. A. B.; KRÜGER, A.; PELlENS, G. C.; BUDAG, J. J.; NADOLNY, M. C. Composição do banco de sementes no solo em áreas de preservação permanente sob diferentes tipos de cobertura. Floresta, Curitiba, v. 43, n. 1, p. 49-58, jan./mar. 2013.

STRECK, E. V. et al. Solos do Rio Grande do Sul. 2. Ed. Porto Alegre: EMATER/RS, 2008. p. 128.

TRES, D. R.; REIS, A. Técnicas nucleadoras na restauração de floresta ribeirinha em área de Floresta Ombrófila Mista, Sul do Brasil. Biotemas, Florianópolis, v. 22, n. 4, p. 59-71, dez. de 2009. 